\title{
EFECTO DE DIFERENTES INTENSIDADES DE PODA SOBRE EL RENDIMIENTO Y CALIDAD DE FRUTA EN ARÁNDANO (Vaccinium corymbosum L. ) cv. BRIGITTA
}

\section{EFFECT OF DIFFERENT PRUNING INTENSITIES ON YIELD AND FRUIT QUALITY OF BLUEBERRY (Vaccinium corymbosum L.) cv. BRIGITTA}

\author{
Pablo Muñoz-Vega ${ }^{1 *}$, Humberto Serri ${ }^{1}$, María Dolores Lopéz ${ }^{1}$, Manuel Faundez ${ }^{1}$, Pia Palma ${ }^{1}$ \\ ${ }^{1}$ Universidad de Concepción, Facultad de Agronomía, Avenida Vicente Méndez 595, Chillán, Chile. \\ * Autor para correspondencia E-mail: pcmunoz@udec.cl
}

\section{RESUMEN}

La presente investigación se realizó en un huerto de arándanos (Vaccinium corymbosum L.) cv. Brigitta de cuarta cosecha, bajo manejo orgánico, situado en la Provincia de Nuble, Chillán, Chile. El objetivo fue determinar el efecto de diferentes intensidades de poda en la producción y calidad de la fruta. Se utilizó un diseño de bloques completos al azar, con cuatro tratamientos y cinco repeticiones, evaluando parámetros vegetativos tales como número de yemas, flores y frutos; parámetros de calidad de la fruta como diámetro ecuatorial y polar, peso promedio de los frutos, número de semillas, sólidos solubles, $\mathrm{pH}$ y acidez titulable, y parámetros de producción. Se determinó que la intensidad de poda afectó la producción por planta y la calidad de la fruta. Los mayores rendimientos se obtuvieron en las plantas con menor intensidad de poda $(\mathrm{P} \leq 0,05)$, pero a su vez esta fruta presentó menor diámetro ecuatorial, menor diámetro polar, menor peso, menor cantidad de semillas, menor porcentaje de sólidos solubles y mayor acidez. El pH de la fruta no presentó diferencias significativas $(\mathrm{P}>0,05)$ en ningún tratamiento. El cv. Brigitta presenta un alto porcentaje de aborto floral, por lo que se debería realizar una poda no tan severa como en otras variedades, dejando un 30-40 \% de carga adicional que una poda normal en otras variedades.

Palabras clave: Arándanos, Vaccinium corymbosum, poda, calidad de fruta.

\section{ABSTRACT}

This research was carried out in a blueberry (Vaccinium corymbosum_L.) orchard cv. Brigitta (fourth harvest) under organic management located in the Province of Nuble (Chillán, Chile). The objective of this work was to determine the effect of different pruning intensities on yield and fruit quality. The experiment was conducted using a randomized complete block design with four treatments and five replications. Parameters related to plant growth (number of buds, flowers and fruits), fruit quality (equatorial and polar diameter, average fruit weight, number of seeds, soluble solids, $\mathrm{pH}$ and titratable acidity), and production were evaluated. The results showed that different pruning intensities resulted in significant differences $(P \leq 0.05)$ in yield and fruit quality. The highest yields were obtained in plants with less severe pruning, but the fruit presented a decrease in equatorial diameter, polar diameter and weight, as well as fewer seeds, lower levels of soluble solids and higher acidity. Fruit $\mathrm{pH}$ showed no significant differences $(\mathrm{P}>0.05)$ between the treatments. As cv. Brigitta presents a high rate of floral abortion, pruning should not be as severe as in other varieties, keeping an additional $30-40 \%$ of fruit load compared to normal pruning in other varieties.

Key words: Blueberries, Vaccinium corymbosum, pruning, fruit quality. 


\section{INTRODUCCIÓN}

El arándano (Vaccinium corymbosum L.) fue introducido en Chile en la década de 1980 para evaluar su potencial como nueva alternativa productiva de fruta para el país (Bañados, 2006). Se introdujo con fines experimentales en el año 1979, sin embargo, la primera plantación comercial se estableció en 1987, con variedades highbush, cerca de La Unión en la Región de Los Lagos (Muñoz, 1993). Actualmente, este cultivo posee una extensión de 14.488 hectáreas bajo manejo convencional y 1.661 has en sistemas orgánicos (ODEPA, 2015).

Los requerimientos de suelo de los arándanos son específicos, puesto que crecen mejor en lugares bien drenados, con luz, suelos franco arenosos con alto contenido de materia orgánica y un pH entre 4,5 y 5,5 (Strik and Finn, 2008). La realización de labores adecuadas permite obtener un mayor rendimiento de este frutal, para lo cual es necesario controlar los factores de variedad (Bryla, 2008), manejo del riego (Bryla et al., 2006; Holzapfel et al., 2015), fertilización con manejo convencional y orgánico, respectivamente (Bryla and Shireman, 2010; Muñoz-Vega et al., 2016) y pH (Ferreyra et al., 2000).

El arándano alto o highbush tiene varias cañas leñosas que surgen a partir de yemas de la corona para formar un arbusto (Gough, 1997). Los brotes de la corona se vuelven leñosos en la segunda temporada de crecimiento (Retamales and Hancock, 2012).

La poda que se realiza inmediatamente después de la plantación tiene por objeto remover las ramas con yemas florales, con lo que mejora el vigor y la sobrevivencia de la planta; se requiere poca poda durante el segundo y tercer año después de plantación (Hanson et al., 2000).

El rendimiento de la planta de arándano está determinado por dos factores principales: el número de frutos por planta y su peso fresco. El número de frutos se estima a partir del número de yemas florales después de la poda y por la densidad de yemas por rama (Salvo et al., 2011).

El arándano solo florece en la madera de un año, y la producción anual se realiza en las zonas más alejadas de las raíces y del centro del arbusto. Esto significa que los nutrientes deben desplazarse una distancia muy larga para llegar a la zona productiva, y además deben alimentar mucho tejido extra no productivo (Retamales and Hancock, 2012). La producción disminuye sustancialmente en cañas de más de cinco años, debido probablemente al transporte de nutrientes a mayor distancia.

La poda es una práctica cultural beneficiosa para el cultivo de arándanos (Williamson et al., 1994) que tiene como objetivo producir consistentemente buenos rendimientos con fruta de alta calidad (Bañados, 2005). También es necesaria para mantener las plantas vigorosas $\mathrm{y}$ un equilibrio adecuado entre crecimiento vegetativo y reproductivo (Retamales and Hancock, 2012). Debido a que el arándano produce más flores que las requeridas para una buena cosecha, la eliminación de algunos brotes durante la poda puede aumentar la calidad de la fruta y también concentrar la maduración del fruto (Strik et al., 2003). La poda anual se recomienda para la estabilidad a largo plazo del rendimiento, ya que si se poda ocasionalmente se produce un balance desigual de ramas muy viejas y jóvenes. Los arbustos de más alto rendimiento tienen alrededor de un 15 a $20 \%$ de cañas jóvenes, $15-20 \%$ de cañas viejas y un $50-70 \%$ de cañas de mediana edad. Las ramas más productivas tienen 2,5 a 3,5 $\mathrm{cm}$ de ancho en su base y 4 a 6 años de edad, pero se necesitan ramas jóvenes de renovación y ramas mayores de apoyo (Retamales and Hancock, 2012).

La poda puede reducir el tamaño de la planta y el rendimiento en la temporada siguiente, pero si se realiza correctamente, permite obtener frutos grandes, maduración temprana y una mayor estabilidad de los rendimientos (Retamales and Hancock, 2012). Una poda fuerte a menudo resulta en la producción de ramas jóvenes vigorosas, que producen frutos de buen tamaño. La poda reduce el número de flores y brotes, por tanto, también el número de bayas, lo que dará como resultado un aumento en el tamaño de las bayas en combinación con un menor rendimiento. Cuando las plantas no se podan suficientemente, el número de brotes florales y bayas es demasiado alto, lo que dará como resultado bayas más pequeñas y de menor calidad (Jansen et al., 1997).

La variedad Brigitta es una planta arbustiva, de crecimiento vertical, con buena ramificación; sus bayas están bien distribuidas sobre toda la planta y presenta muy buena producción. La fruta es una baya de tamaño medio, con sabor dulce, la maduración es de media estación, presenta una cosecha compacta y la fruta presenta buena calidad después de 6 a 7 semanas de almacenamiento (Bal et al., 2006).

Las bajas temperaturas invernales en las regiones frías a menudo causan graves daños a los arándanos en sus flores, yemas y brotes jóvenes (Kovaleski et al., 2015). Heladas de primavera comúnmente dañan los brotes de flor de todas las especies de arándanos. Los cultivares con fechas tardía de floración tienden a sufrir menos daños por helada que los que florecen antes, porque las heladas son menos comunes y 
la etapa de desarrollo floral coincide con la yema relativamente fortalecida (Lin y Pliszka, 2003). En Chile, las heladas durante septiembre y octubre a veces destruyen las flores y frutos de arándanos (Lyrene y Muñoz, 1997).

El efecto de la poda en arándanos ha sido estudiado por numerosos autores en los últimos años (Strik et al., 2003); por otra parte, la intensidad de poda, área foliar e intercambio de gases en hoja y la evaluación de carga de fruta como un predictor de peso fresco, \% de materia seca y la concentración de azúcar en la variedad Brigitta fue estudiado por Jorquera-Fontana et al. (2014).

En la Región del Biobío el cv. Brigitta presenta menor fructificación que el resto de las variedades de arándanos, Independiente del tamaño y numero de yemas florales que posea, siempre tiende a fructificar un 30 a $40 \%$ menos de lo estimado, lo que podría ser un problema de cuaja durante la floración, ocasionado por temperaturas bajo cero o mala polinización (Muñoz y Serri. Datos no publicados).

El presente estudio tuvo como objetivo evaluar el efecto de diferentes intensidades de poda sobre el rendimiento y calidad de fruta de arándano variedad Brigitta, en la provincia de Nuble, Chile.

\section{MATERIALES Y MÉTODOS}

\section{Ubicación del ensayo}

El estudio se realizó durante la temporada 2013-2014 en un huerto orgánico de arándano cultivar Brigitta en cuarta cosecha, ubicado en la Estación Experimental "El Nogal", perteneciente a la Facultad de Agronomía de la Universidad

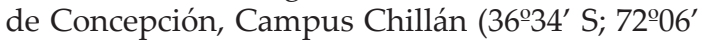
O; a 144 m.s.n.m.). El clima es tipo mediterráneo húmedo, con un régimen térmico templado, con una temperatura media anual de $13,3^{\circ} \mathrm{C}$ y una precipitación media anual entre 1.000 a $1.200 \mathrm{~mm}$ año ${ }^{-1}$ (CIREN, 1999). El suelo del sitio experimental corresponde a una clasificación medial, amorphic, thermic humic haploxerands. (Stolpe, 2006), con buen drenaje y disponibilidad de agua de riego. El huerto posee un marco de plantación de $3,0 \mathrm{~m}$ por $1,0 \mathrm{~m}$, entre y sobre hilera, respectivamente, y el sistema de riego es por goteo con un caudal de 2,1 $\mathrm{L} \mathrm{h}^{-1}$.

\section{Tratamientos}

Se evaluaron 4 tratamientos de poda (Tabla 1 ), para ello, se seleccionaron 20 plantas homogéneas $\mathrm{y}$ con idéntico desarrollo vegetativo, edad y número total de yemas florales. Además en cada tratamiento se seleccionaron 4 ramillas según los puntos cardinales. Cada ramilla se marcó con una cinta y se registró el número de yemas florales de la rama y la coordenada. La poda se realizó en el mes de agosto.

\section{Evaluaciones}

Número de yemas florales. Una vez realizada la poda se registró el número de yemas de las 4 ramillas seleccionadas de cada planta.

Flores. Se registró semanalmente el número de flores de las ramillas seleccionadas.

Aborto floral. Semanalmente se controló el número de flores, y la caída, ya sea por condiciones climáticas (temperatura), plagas y/o enfermedades.

Diámetro ecuatorial y polar. Se tomó una muestra representativa de 10 frutos de las cuatro ramillas seleccionadas por tratamiento, y se registró el diámetro ecuatorial y polar de cada uno de los frutos, esto labor se realizó en cada cosecha utilizando un pie de metro digital, marca Stainless Hardened.

Peso promedio del fruto. Se determinó pesando con una balanza digital 100 frutos al azar por cada tratamiento.

Producción por planta. La producción por planta se obtuvo pesando el total de la fruta cosechada por planta, mediante el uso de una balanza digital.

Número de semillas por fruto. Se determinó contabilizando el número de semillas de 5 frutos

Tabla 1. Número de yemas por planta que se dejaron en cada tratamiento de poda realizados en arándanos variedad Brigitta en la Provincia de Ñuble.

Table 1. Number of buds left per plant after each pruning intensity treatment in 'Brigitta' blueberries, Province of Nuble.

\begin{tabular}{ccc}
\hline Tratamientos & \% de yemas & $\mathbf{N}^{\circ}$ de yemas/planta \\
\hline 1 & 25 & 125 \\
2 & 50 & 250 \\
3 & 75 & 375 \\
4 & 100 & 500 (Sin poda) \\
\hline
\end{tabular}


seleccionados al azar de cada ramilla previamente elegida en los cuatro puntos cardinales para cada tratamiento.

Sólidos solubles. Se determinó con un refractómetro en la tercera cosecha a partir del jugo o zumo del total de la planta cosechada; se realizaron 3 mediciones por muestra, sacando un promedio de los grados Brix.

pH. Se determinó con un potenciómetro $(\mathrm{pH}-$ metro) en la tercera cosecha a partir del jugo o zumo del total de los frutos de la planta cosechada. Se realizaron 3 mediciones por muestra.

Acidez titulable. Se determinó en la tercera cosecha a partir del jugo o zumo del total de la cosechada. Se realizaron 3 mediciones por muestra. La acidez titulable y su cálculo se realizó según el protocolo utilizado por Connor et al. (2002) con modificaciones para arándano (teniendo en cuenta el ácido mayoritario para el arándano que es el ácido cítrico):

$$
\% \text { Acidez }=\left(\frac{A \times B \times C}{D}\right) \times 100
$$

dónde: $\mathrm{A}=$ cantidad de base o $\mathrm{NaOH}$ (hidróxido de sodio) gastado, en $\mathrm{mL} ; \mathrm{B}=$ normalidad de la base $(\mathrm{NaOH})$ usada en la titulación $(0,1 \mathrm{~N}) ; \mathrm{C}=$ peso equivalente expresado en gramos de ácido predominante de la fruta (64 g, para arándanos es el ácido cítrico); y $\mathrm{D}$ = peso de la muestra en gramos.

\section{Diseño experimental y análisis estadístico}

El diseño experimental fue de bloques completos al azar, con cuatro tratamientos y 5 repeticiones. Se realizó un análisis de varianza $(\alpha$ $=0,05)$, y una prueba de comparación de medidas de LSD Fisher con una significancia de $95 \%$ ( $\mathrm{P} \leq$ $0,05)$ para los parámetros de fenología y calidad. Para el análisis estadístico se utilizó el programa estadístico InfoStat (Balzarini et al., 2008).

\section{RESULTADOS Y DISCUSIÓN}

\section{Número de flores y frutos}

El número de flores y frutos se presenta en la Tabla 2. Se registró el número de yemas el día 14 de septiembre, luego a partir de la semana del 30 de septiembre se monitoreó y registró el número de flores. La floración se concentró en dos semanas, desde el 30 de septiembre hasta el 14 de octubre, ocurriendo la plena floración la semana del 30 de septiembre al 7 de octubre. Esto concuerda con lo expuesto con Buzeta (1997) que menciona que el periodo de plena floración normalmente dura 7 días. Además, durante el periodo de floración se produjo aborto floral, lo que se registró en las cuatro ramas seleccionadas por tratamiento. Se observó una cuaja menor a la cantidad de flores registradas al inicio del ensayo (Tabla 2). La disminución del porcentaje de cuaja se atribuyó por daño de las bajas temperaturas registrada. Los datos aportados por la Estación Meteorológica de la Universidad de Concepción, indican que durante el periodo del 28 de septiembre al 4 de octubre la temperatura mínima fluctuó entre $\operatorname{los}-2,3^{\circ} \mathrm{C}$ y $0^{\circ} \mathrm{C}$. Estas temperaturas son perjudiciales para los estados de yema hinchada y floración, en los cuales se encontraba la planta en este periodo. Con respecto a esto, Spiers (1978), Cappiello y Dunhan et al. (1994) y Warmund et al. (2008) encontraron una gran relación entre el grado de desarrollo de la yema floral y la temperatura que daña la flor. En este estudio las yemas florales visiblemente abultadas pero con flores totalmente cerradas, soportaron temperaturas de $-6^{\circ} \mathrm{C}$. Los brotes en que las flores eran distinguibles individualmente murieron a $-4{ }^{\circ} \mathrm{C}$. Sin embargo las flores que se habían separado claramente, pero con la corola no expandida y cerrada, murieron a $\operatorname{los}-2^{\circ} \mathrm{C}$, en cambio las flores completamente abiertas fueron severamente dañadas a $0^{\circ} \mathrm{C}$. Esto explicaría la

Tabla 2. Yemas florales, flores, frutos, porcentaje de cuaja y frutos por planta, en distintas intensidades de poda, en la Provincia de Nuble.

Table 2. Number of flower buds, flowers and fruits, and percentage of fruits set and harvested under different intensities of pruning, Province of Nuble.

\begin{tabular}{lcccccc}
\hline Tratamientos & $\begin{array}{c}\text { Número de } \\
\text { yemas florales }\end{array}$ & $\begin{array}{c}\text { Número de } \\
\text { flores }\end{array}$ & $\begin{array}{c}\text { Número de } \\
\text { frutos }\end{array}$ & $\begin{array}{c}\text { Porcentaje } \\
\text { de frutos } \\
\text { cuajados } \\
\mathbf{( \% )}\end{array}$ & $\begin{array}{c}\text { Número } \\
\text { de } \\
\text { cosechados }\end{array}$ & $\begin{array}{c}\text { Porcentaje de } \\
\text { frutos } \\
\text { cosechados } \\
\text { (\%) }\end{array}$ \\
\hline $125 \%$ & 12 & 95 & 78 & 82 & 63 & 66 \\
$250 \%$ & 23 & 109 & 85 & 77 & 76 & 70 \\
$375 \%$ & 26 & 98 & 80 & 81 & 71 & 72 \\
4 Sin Poda & 25 & 150 & 124 & 82 & 105 & 70 \\
\hline
\end{tabular}


disminución en el número de flores en aquellas que se encontraban completamente abiertas en el periodo de plena floración.

Por otra parte, Retamales y Hancock (2012) señalan que además del daño por helada hay otros factores que afectan la floración, señalando que todas las variedades de arándano requieren un polinizador, siendo la densidad de abejas un factor crítico en la determinación de la cuaja. En este contexto, Scherm et al. (2001) mencionan que una vez que el polen viable es depositado en el estigma, la polinización exitosa está determinada por tres factores: la receptividad de la flor, el grado de auto-fertilidad y la disponibilidad de recursos para la maduración del fruto.

En el primer periodo de desarrollo de los frutos se registró una disminución en el número de frutos por planta (Tabla 2). Se descartó el daño por helada, dado que los datos climatológicos de la Universidad de Concepción no indican temperaturas inferiores a cero grados Celsius durante ese periodo.

\section{Diámetro ecuatorial y polar}

El diámetro ecuatorial de la baya varió significativamente $(\mathrm{P} \leq 0,05)$ con la intensidad de poda (Tabla 3), observándose un mayor diámetro ecuatorial en el tratamiento de mayor intensidad de poda, correspondiente a 14,55 mm, y el menor diámetro ecuatorial se obtuvo en el tratamiento de menor intensidad de poda, correspondiente a $12,60 \mathrm{~mm}$.

Buzeta (1997) mencionó que dependiendo de la especie y cultivar, el diámetro ecuatorial puede variar entre 0,7 a $1,5 \mathrm{~cm}$, lo cual coincide con los resultados obtenidos en este estudio, superando ese parámetro en los tratamientos de mayor intensidad de poda.

La norma de calidad establecida por el Chilean Blueberry Comitte (2013) cataloga como defecto menor a aquella fruta que posee un diámetro ecuatorial inferior a $10 \mathrm{~mm}$, lo que no se presentó en éste estudio, lo que indica que la variedad Brigitta aunque no sea podada, cumple con los requisitos de exportación.

El diámetro polar de la baya varió significativamente $(P \leq 0,05)$ con la intensidad de la poda, obteniendo un mayor diámetro en los tratamientos de mayor intensidad de poda, correspondientes a $11,54 \mathrm{~mm}$., y el menor diámetro se obtuvo en los tratamientos de menor intensidad de poda, correspondientes a $9,60 \mathrm{~mm}$. Los tratamientos con el total de yemas (500 yemas) y de menor intensidad de poda (375 yemas) no presentaron diferencias significativas $(P \geq 0,05)$, por lo que una poda ligera produciría un efecto similar a no podar.

\section{Peso promedio de los frutos}

En la Fig. 1 se presentan los resultados del peso promedio de los frutos. El mayor peso por fruto se obtuvo en los tratamientos de mayor intensidad de poda, correspondientes a 1,93 $\mathrm{g}_{\text {fruto }}{ }^{-1}$. Gautier et al. (2001) en un estudio en tomates, mencionan que a menor carga frutal hay mayor disponibilidad de agua y carbohidratos, lo que produce un mayor peso de los frutos. El menor peso promedio por fruto se obtuvo en los tratamientos de menor intensidad de poda correspondientes a $1,20 \mathrm{~g}_{\text {fruto }}^{-1}$. Por otra parte, Siefker and Hancock (1986) en un estudio realizado con nueve cultivares de arándano alto, determinaron que en la mayoría de las variedades, al aumentar el número de bayas por caña, disminuye el peso de la baya, lo que también se observó en este estudio.

Pedreros et al. (2007) mencionan que en condiciones de suelo trumao en Ñuble, la presencia de malezas en el periodo de crecimiento, floración y fructificación de las plantas de arándano, reducen el rendimiento del fruto, lo que explicaría el menor peso promedio del fruto observado en este estudio. Pedreros y Rebolledo (2007) mencionan que las malezas reducen el

Tabla 3. Efecto de cuatro intensidades de poda sobre el diámetro ecuatorial y diámetro polar en arándanos variedad Brigitta en la Provincia de Ñuble.

Table 3. Effect of four pruning intensities on the equatorial diameter and polar diameter of 'Brigitta' blueberries, Province of Nuble.

\begin{tabular}{clcc}
\hline Tratamientos & Diámetro ecuatorial & Diámetro polar \\
& & mm & \\
\hline 1 & $25 \%$ & $14,55 \mathrm{a}$ & $11,54 \mathrm{a}$ \\
2 & $50 \%$ & $13,69 \mathrm{~b}$ & $11,00 \mathrm{~b}$ \\
3 & $75 \%$ & $13,17 \mathrm{c}$ & $9,93 \mathrm{c}$ \\
4 & Sin poda & $12,60 \mathrm{~d}$ & $9,60 \mathrm{c}$ \\
\hline
\end{tabular}

Letras distintas indican que hay diferencias significativas, según test de LSD Fisher $(P \leq 0,05)$. 


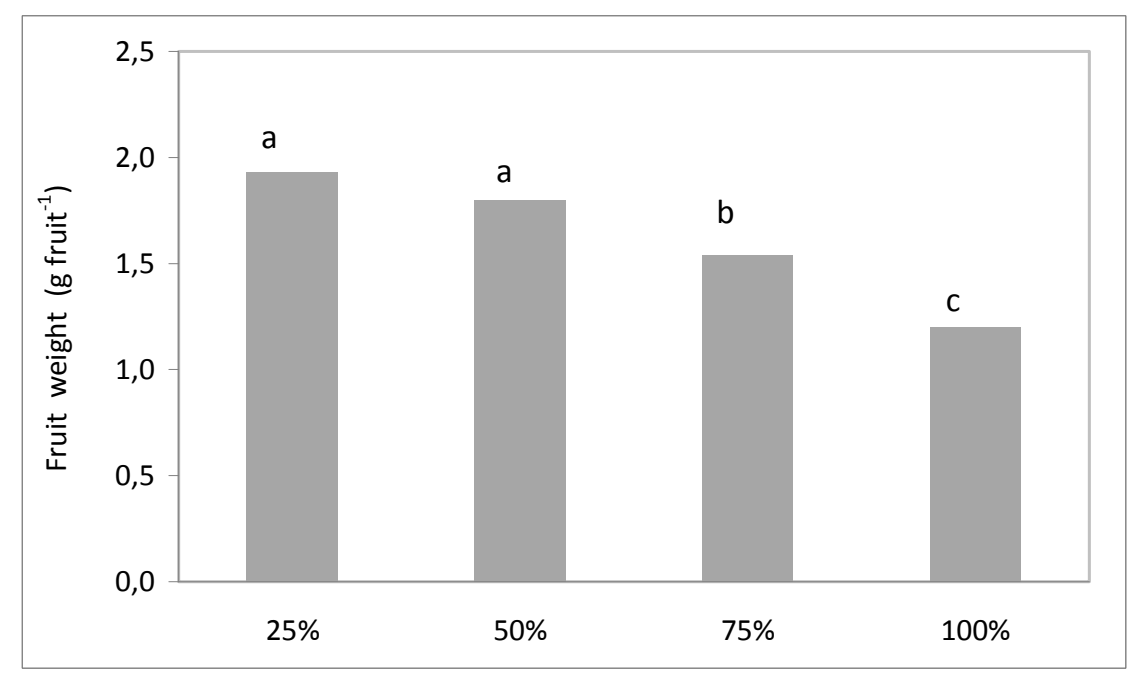

Fig. 1. Peso promedio de frutos de arándano variedad Brigitta en la Provincia de Nuble, con distintas intensidades de poda.

Fig. 1. Average fruit weight of 'Brigitta' blueberries under different pruning intensities, Province of Nuble.

Las letras distintas indican que hay diferencias significativas, según test de LSD Fisher $(\mathrm{P} \leq 0,05)$.

rendimiento debido a la competencia por agua, nutrientes, luz y espacio. En el caso específico de los arándanos, hay malezas que florecen en el mismo periodo del cultivo, por lo que además de lo indicado, compiten por insectos polinizantes.

\section{Producción por planta}

Se obtuvieron diferencias significativas ( $\mathrm{P} \leq$ 0,05 ) en producción total por planta para todas las intensidades de poda (Tabla 4), obteniendo la menor producción por planta en el tratamiento de mayor intensidad de poda, correspondiente a 1056,11 g planta $^{-1}$, y la mayor producción por planta se obtuvo en el tratamiento de menor intensidad de poda, correspondiente a 2167,67 g planta $^{-1}$, siendo mayor en un 51,3\%.

No se observaron diferencias significativas $(\mathrm{P}>0,05)$ para la repetición, la orientación y la interacción entre el tratamiento y la orientación.

Con los resultados obtenidos con las diferentes intensidades de poda, se podría optar por no podar esta variedad, para aumentar los rendimientos y la producción, y así obtener mayores beneficios económicos. Sin embargo, Strik et al. (2003) mencionan que producir demasiada fruta en las plantas jóvenes reduce el rendimiento y crecimiento posterior, lo que podría ser perjudicial. También esta fruta podría ser de menor calidad, como lo indican Léchaudel et al. (2005), que mencionan que aunque un gran número de fruta por planta es deseable para lograr un mayor rendimiento por unidad de superficie, la competencia por asimilados entre la fruta puede reducir el área total del dosel, lo que conduciría a una calidad de fruta inferior.

La fruta de mayor calibre y de mayor peso que se obtiene con los tratamientos de mayor intensidad de poda no logra alcanzar una mayor producción que los tratamientos con menor intensidad de poda con fruta de menor calibre y menor peso. Jorquera-Fontana et al. (2014) indican que a mayor intensidad de poda, aumenta el peso de la baya, pero esta no compensa la pérdida de rendimiento por planta, lo que concuerda con los resultados obtenidos en este estudio.

La poda tiene un impacto importante en la producción. Al respecto, Siefker y Hancock (1986) y Strik y Finn (2008), indican que la producción en arándano está fuertemente determinada por el número de frutos que producen las ramas y el peso de los frutos, lo que podría explicar el menor rendimiento en los tratamientos con podas más severas.

\section{Número de semillas}

Se observaron diferencias significativas $(\mathrm{P} \leq$ $0,05)$ en el número de semillas en los tratamientos con mayor intensidad de poda (Tabla 4), obteniendo mayor número de semillas el tratamiento con mayor intensidad de poda, siendo un $66,23 \%$ mayor en relación al tratamiento sin poda. No se observaron diferencias significativas $(P>0,05)$ en el tratamiento de 375 yemas, y el tratamiento sin poda. Retamales y Hancock 
Tabla 4. Efecto de cuatro intensidades de poda sobre el peso promedio de los frutos, producción por planta y número de semillas en arándanos variedad Brigitta en la Provincia de Nuble.

Table 4. Effect of four pruning intensities on average fruit weight, yield per plant and number of seeds in 'Brigitta' blueberries, Province of Ñuble.

\begin{tabular}{|c|c|c|c|}
\hline Tratamientos & $\begin{array}{c}\text { Peso promedio } \\
\text { frutos } \\
\left(\mathrm{g} \text { fruto }^{-1}\right)\end{array}$ & $\begin{array}{c}\text { Producción por } \\
\text { planta } \\
\left(\mathrm{g} \text { planta }{ }^{-1}\right)\end{array}$ & $\begin{array}{c}\text { Número de } \\
\text { semillas }\end{array}$ \\
\hline $125 \%$ & $1,93 \mathrm{a}$ & $1056,11 \mathrm{a}$ & $24,70 \mathrm{a}$ \\
\hline $250 \%$ & $1,80 \mathrm{a}$ & $1451,81 \mathrm{~b}$ & $20,60 \mathrm{~b}$ \\
\hline $375 \%$ & $1,54 \mathrm{~b}$ & $1612,39 \mathrm{c}$ & $17,06 \mathrm{c}$ \\
\hline 4 Sin poda & $1,20 \mathrm{c}$ & $2167,67 \mathrm{~d}$ & $16,36 \mathrm{c}$ \\
\hline
\end{tabular}

Las letras distintas indican que hay diferencias significativas, según test de LSD Fisher $(\mathrm{P} \leq 0,05)$.

(2012) mencionan que el arándano alto y ojo de conejo tienen más de 110 óvulos por fruto, pero el número de semillas desarrolladas rara vez supera la mitad de ese número. En ésta investigación el número de semillas fue mucho menor, donde la mayor cantidad de semillas fue de 24,7 semillas.

Los resultados obtenidos no concuerdan con lo señalado por Mackenzie (1997), quien observó que el número de semillas por fruto en cuatro cultivares de "arándano alto" varió entre 46 y 88 semillas, señalando además que el número de semillas por fruto varía de un año a otro, por la influencia de las condiciones climáticas que afectan la duración de la floración y la actividad de los polinizadores.

Por otra parte, Buzeta (1997) indica que hay una correlación entre el contenido de semillas y el tamaño del fruto, es decir que a mayor número de semillas será mayor el tamaño del fruto En este estudio se obtuvo la mayor cantidad de semillas en los tratamientos de mayor intensidad de poda, que tuvieron frutos de mayor calibre. En esta investigación para la variedad Brigitta el número de semillas varió entre 16 a 32 semillas por fruto.

\section{Sólidos solubles, pH y acidez titulable.}

La poda afectó significativamente $(\mathrm{P} \leq 0,05)$ el porcentaje de sólidos solubles, observándose una mayor concentración en los tratamientos de mayor intensidad de poda, correspondientes a $12,22 \%$, y el menor porcentaje se obtuvo en los tratamientos sin poda correspondientes a $10,66 \%$ (Tabla 5).

En un estudio realizado por Jorquera-Fontana et al. (2014) sobre el efecto de la intensidad de la poda en la respuesta de plantas de arándano variedad Brigitta, encontraron que una poda ligera podría proporcionar el potencial para un mayor rendimiento en plantas de 4 y 5 años, en condiciones de huertos del sur de Chile, pero el fruto presenta un menor porcentaje de sólidos solubles, lo cual concuerda con este estudio. Según Retamales y Hancock (2012) esto confirma que la intensidad de poda tiene un impacto importante en la calidad de frutas de arándano. Además, Gündüz et al. (2015) indican que en un estudio donde se evaluó el efecto del cultivar y la temporada en la calidad de la fruta para arándanos alto y ojo de conejo, el cultivar Brigitta presentó un porcentaje de sólidos solubles de 12,9 y $14,2 \%$, para la temporada 2010 y 2011, lo que coincide con lo obtenido en este estudio.

En cuanto al $\mathrm{pH}$, este no presentó diferencias significativas $(\mathrm{P} \geq 0,05)$ (Tabla 5). Los valores obtenidos fluctuaron de 2,90 a 2,94, valores parecidos a los reportados por Gündüz et al. (2015)

La acidez titulable de la fruta presentó diferencias significativas $(\mathrm{P} \leq 0,05)$ (Tabla 5), observándose la mayor acidez en el tratamiento sin poda $(1,05)$ y la menor acidez se obtuvo en los tratamientos de mayor intensidad de poda $(0,75)$. Para la variedad Brigitta, Gündüz et al. (2015) obtuvieron un valor de acidez titulable 0,9 para la temporada 2010 y 2011, valor que se mantuvo para ambos años; en este caso la acidez titulable varió en los distintos tratamientos y presento un rango de 0,76 a 1,05. Además, se observó una relación inversa entre los sólidos solubles y la acidez titulable, donde a mayor porcentaje de sólidos soluble menor fue la acidez titulable. Wang and Zheng (2005) y Wang et al. (2008) sñalaron que a medida que el fruto madura en la planta disminuye la acidez titulable, mientras que los sólidos solubles aumentan, lo que explicaría lo que se observó en esta investigación. 
Tabla 5. Efecto de cuatro intensidades de poda sobre los sólidos solubles, pH y acidez titulable en arándanos variedad Brigitta en la Provincia de Ñuble.

Table 5. Effect of four pruning intensities on soluble solids, $\mathrm{pH}$ and titratable acidity in 'Brigitta' blueberries, Province of Ñuble.

\begin{tabular}{ccccc}
\hline Tratamientos & $\begin{array}{c}\text { Sólidos solubles } \\
\text { \% }\end{array}$ & pH & $\begin{array}{c}\text { Acidez Titulable } \\
\%\end{array}$ \\
\hline 1 & $25 \%$ & $12,22 \mathrm{a}$ & $2,93 \mathrm{a}$ & $0,76 \mathrm{a}$ \\
2 & $11,65 \mathrm{~b}$ & $2,90 \mathrm{a}$ & $0,86 \mathrm{~b}$ \\
3 & $75 \%$ & $11,42 \mathrm{~b}$ & $2,93 \mathrm{a}$ & $0,93 \mathrm{c}$ \\
4 & Sin poda & $10,66 \mathrm{c}$ & $2,94 \mathrm{a}$ & $1,05 \mathrm{~d}$ \\
\hline
\end{tabular}

Las letras distintas indican que hay diferencias significativas, según test de LSD Fisher $(P \leq 0,05)$.

\section{CONCLUSIONES}

De acuerdo a los resultados obtenidos y a las condiciones en que se realizó la investigación, se puede concluir que:

A mayor intensidad de poda se obtienen menores rendimientos pero la fruta presenta mayor calibre, cantidad de semillas y peso de fruto.

En cuanto a los índices de calidad, a mayor intensidad de poda mayor es el porcentaje de sólidos solubles y menor valor de acidez titulable.

El cv. Brigitta presenta un alto porcentaje de aborto floral, por lo que se debería realizar una poda no tan severa como en otras variedades, dejando un $30-40 \%$ más de carga que una poda normal en otra variedad.

\section{LITERATURA CITADA}

Bal, J.J.M., J.M.T. Balkhoven, and G. Peppelman. 2006. Results of testing highbush blueberry cultivars in The Netherlands. Acta Hortic. 715:157-162.

Balzarini, M.G., M. Gonzalez, M. Tablada, F. Casanoves, J.A. Di Rienzo, y C.W. Robledo. 2008. InfoStat: sofware estadístico. Manual del usuario. Versión 2008. 336 p. Brujas, Córdoba, Argentina.

Bañados, P. 2005. Claves para la poda de arándanos: temporada de invierno. Agron. For. UC 25:28-31.

Bañados, M.P. 2006. Blueberry production in South America. Acta Hortic. 715:165-172.

Bryla, D. 2008. Water requirements of young blueberry plants irrigated by sprinklers, microsprays and drip. Acta Hortic. 792:135-140.
Bryla, D., J. Gartung, and B.C. Strik. 2006. Evaluation of irrigation methods for highbush blueberry. I. Growth and water requirements of young plants. HortScience 46(1):95-101.

Bryla, D.R., and A.D. Shireman. 2010. Effects of method and level of nitrogen fertilizer application on soil $\mathrm{pH}$., electrical conductivity, and availability of ammonium and nitrate in blueberry. Acta Hortic. 868:95102.

Buzeta, A. 1997. Chile: berries para el 2000. 132 p. Fundación Chile, Santiago, Chile.

Cappiello, P.E., and S.W. Dunham. 1994. Seasonal variation in low-temperature tolerance of Vaccinium angustifolium Ait. HortScience 29(4):302-304.

Chilean Blueberry Committe. 2013. Norma de calidad arándano fresco de exportación v.02 Disponible en Chilean Blueberry. https://www.yumpu.com/es/document/ view/47062951/norma-de-calidad-cbbcv02-comite-de-arandanos/4 (Consulta 28 noviembre 2016)

CIREN. 1999. Descripciones de suelos materiales y símbolos: Estudio Agrológico VIII Región. Publicación CIREN № 121. 583 p. Centro de Información de Recursos Naturales (CIREN), Santiago, Chile.

Connor, A., J. Luby, J. Hancok, S. Berkheimer, and J. Hanson, J. 2002. Changes in fruit antioxidant activity among blueberry cultivars during cold-temperature storage. Agric. Food Chem. 50(4):893-898.

Ferreyra, R., J. Peralta, A. Sadzawka, C. Muñoz, and J. Valenzuela. 2000. Effect of substrate and irrigation water acidification on the nutrition, growth, and yield of rabbiteye blueberries (Vaccinium ashei Reade). Agricultura Técnica 61:452-458. 
Gautier, H., S. Guichard, and M. Tchamitchian. 2001. Modulation of competition between fruits and leaves by flower pruning and water fogging, and consequences on tomato leaf and fruit growth. Ann. Bot. 88(4):645-652.

Gough, R.E. 1997. Blueberries - North and south. J. Small Fruit Vitic. 4(1-2):71-106.

Gündüz, K., S. Serçe, and J.F. Hancock. 2015. Variation among hightbush and rabbiteye cultivars of blueberry for fruit quality and phytochemical characteristics. J. Food Compos. Anal. 38:69-79.

Hanson E., J. Hancock, D.C. Ramsdell, A. Schilder, G. VanEe, and R. Ledebuhr. 2000. Sprayer type and pruning affect the incidence of blueberry fruit roots. HortScience 35(2):235238.

Holzapfel E., J. Jara, and A.M. Coronata. 2015. Number of drip laterals and irrigation frequency on yield and exportable fruit size of highbush blueberry grown in a sandy soil. Agricultural Water Management 148:207212.

Jansen, W.A.G.M. 1997. Pruning of highbush blueberries. Acta Hortic. (446):333-336.

Jorquena-Fontana, E., M. Alberdi, and N. Franck. 2014. Pruning severity affects yield, fruit load and fruit and leaf traits of 'Brigitta' blueberry. J. Soil Sci. Plant Nutr. 14(4):855-868.

Kovaleski P., G. Williamson, B. Casamali, and R. Darnell. 2015. Effects of timing and intensity of summer pruning on vegetative traits of two southern highbush blueberry cultivars. HortScience 50(1):68-73.

Léchaudel, M., J. Joas, Y. Caro, M. Génard, and M. Jannoyer. 2005. Leaf:fruit ratio and irrigation supply affect seasonal changes in minerals, organic acids and sugars of mango fruit. J. Sci. Food Agric. 85(2):251-260.

Lin, W., and K. Pliszka. 2003. Comparison of spring frost tolerance among different highbush blueberry (Vaccinium corymbosum L.) cultivars. Acta Hortic. 626:337-341.

Lyrene, P.M., and C. Muñoz. 1997. Blueberry production in Chile. J. Small Fruit Vitic. 5(1):1-20.

MacKenzie, K.E. 1997. Pollination requirements of three highbush blueberry (Vaccinium corymbosum L.) cultivars. J. Am. Soc. Hortic. Sci. 122(6):891-896.

Muñoz, C. 1993. Overview of the blueberry industry in South America. Acta Hortic. (346):27-32.
Muñoz-Vega, P., H. Paillán, H. Serri, D. Donnay, C. Sanhueza, E. Merino, et al. 2016. Effects of organic fertilizers on the vegetative, nutritional, and productive parameters of blueberries 'Corona', 'Legacy', and 'Liberty'. Chilean Journal of Agricultural Research 76(2):201-2012.

ODEPA. 2015. Superficies de frutales por región [en línea] Oficina de Estudios y Políticas Agrarias (ODEPA), Chile. Disponible en http://www.odepa.cl/superficie-de-frutalespor-region-2/ (Consulta 7 octubre 2015).

Pedreros, A., y C. Rebolledo. 2007. Malezas en arándanos: efectos en el rendimiento y alternativas de manejo sustentable. Informativo $\mathrm{N}^{\circ}$ 105. INIA Quilamapu, Chillán, Chile.

Pedreros, A., P. Grau, M. Lagos, y C. Rebolledo. 2007. Malezas en arándano. Tierra Adentro (75):35-37.

Retamales, J.B., and J.F. Hancock. 2012. Blueberries. 323 p. Crop Production Science in Horticulture $\mathrm{N}^{\circ}$ 21. CABI, Wallingford, UK.

Salvo, S., C. Muñoz, J. Ávila, J. Bustos, E. Cariaga, C. Silva, et al. 2011. Sensitivity in the estimation of parameters fitted by simple linear regression models in the ratio of blueberry buds to fruits in Chile using percentage counting. Sci. Hortic. 130(2):404409.

Scherm, H., D.S. Nesmith, D.L. Horton, and G. Krewer. 2001. A survey of horticultural and pest management practices of the Georgia blueberry industry. Small Fruits Rev. 1(4):1728.

Siefker, J.A., and J.F. Hancock. 1986. Yield component interactions in cultivars of the highbush blueberry. J. Am. Soc. Hortic. Sci. 111(4):606-608.

Spiers, J.M. 1978. Effect of stage of bud development on cold injury in rabbiteye blueberry. J. Am. Soc. Hortic. Sci. 103(4):452455.

Stolpe, N. 2006. Descripciones de los Principales Suelos de la VIII Región de Chile. 84 p. Departamento de Suelos y Recursos Naturales, Facultad de Agronomía, Universidad de Concepción, Chillán, Chile.

Strik, B., G. Buller, and E. Hellman. 2003. Pruning severity affects yield, berry weight, and hand harvest efficiency of highbush blueberry. HortScience 38(2):196-199. 
Strik, B.C., and C.E. Finn. 2008. Blueberry cultivars for Oregon. Oregon State University, USA. Available at http://ir.library.oregonstate. edu/xmlui/bitstream/handle/1957/18937/ ec1308-e.pdf?sequence $=4 \quad$ (Accessed 7 October 2015).

Wang Y, and W. Zheng. 2005 Preharvest application of methyl jasmonate increases fruit quality and antioxidant capacity in raspberries. International Journal of Food Science and Technology 40:187-195.

Wang Y, L. Bowman, M. Ding. 2008. Methyl jasmonate enhances antioxidant activity and flavonoid content in blackberries (Rubus sp.) and promotes antiproliferation of human cancer cells. Food Chemistry 107:1261-1269.

Warmund, M.R., P. Guinan, and G. Fernandez. 2008. Temperatures and cold damage to small fruit crops across the eastern United States associated with the April 2007 freeze. HortScience 43(6):1643-1647.

Williamson, J.G., F.S. Davies, and P.M. Lyrene. 1994. Pruning blueberry plant in Florida [on line]. University of Florida. IFAS Extension, USA. Available at <http://dixie.ifas.ufl.edu/ pdfs/gardening/blueberry_prune.pdf $>$. (Accessed 14 May 2015). 